\title{
Suicide Probability in Adolescents With a History of Childhood Maltreatment: The Role of Non-Suicidal Self-Injury, Emotion Regulation Difficulties, and Forms of Self-Criticism
}

\author{
Hamid Khanipour, ${ }^{1,}$ Mitra Hakim shooshtari, ${ }^{2}$ and Reza Bidaki ${ }^{3}$ \\ ${ }^{1}$ Institute of Educational, Psychological and Social Research, Kharazmi University, Tehran, IR Iran \\ ${ }^{2}$ Iran University of Medical Sciences, Tehran Psychiatric Institute and Mental Health Research Center, Tehran, IR Iran \\ ${ }^{3}$ Department of Psychiatry, Research Center of Addiction and Behavioral Sciences, Research Center of Diabetes, Shahid Sadoughi University of Medical Sciences, Yazd, IR Iran \\ "Corresponding author: Hamid Khanipour, Institute of Educational, Psychological and Social Research, Kharazmi University, Tehran, IR Iran. Tel: +98-9118449609, Fax: \\ +98-2166551515, E-mail: Khanipur.hamid@gmail.com
}

Received 2014 September 26; Revised 2015 April 18; Accepted 2015 May 26.

\begin{abstract}
Background: Suicidal attempt and non-suicidal self-injury are very common in adolescents with a history of childhood maltreatment. By identifying correlates of these kinds of high-risk behaviors, it is possible to prevent and decrease completed suicide.

Objectives: The aims of this study were: 1) to compare adolescents with a history of childhood maltreatment with non-suicidal self injury (NSSI) or past suicidal attempt in terms of suicide probability, and 2) to investigate the association between NSSI, forms of self-criticism, emotion regulation difficulties, and suicide probability.

Patients and Methods: Participants were 169 adolescents living in Iranian social welfare centers who had a history of childhood maltreatment. The Suicide Probability Scale, Difficulties in Emotion Regulation Scale, Forms of Self-criticism, and the Non-Suicidal Self injury (NSSI) checklist were used for assessment.

Results: Adolescents with NSSI and suicidal attempts had higher rates of suicide ideation than adolescents with NSSI-only (P < 0.05). Feelings of inadequacy, self-hatred, difficulty with impulse control, and frequency of NSSI can predict $50 \%$ variance of suicide probability $(\mathrm{P}<0.001)$.

Conclusions: Adolescents with histories of suicidal attempts and NSSI, compared with adolescents with NSSI-only, were more prone to suicide. Self-criticism, poor impulse control, and the frequency of NSSI were the main risk factors associated with suicide probability in adolescents with a history of childhood maltreatment.
\end{abstract}

Keywords: Self-Injurious Behavior, Suicide, Emotional Disturbances, Self-Criticisms

\section{Background}

According to reports of USA health statics center in 2009 , suicide was the third leading cause of death for those ages 10 to 19 years in the USA (1). Also, the findings of a study conducted in Iran showed that adolescents are more at risk for suicidal attempts than non-adolescents (2). The rates of suicidal attempts and other self-harming behaviors in adolescents with a history of childhood maltreatment are higher than general population (3). While today we have more information about suicidal attempt than ever, information about suicide probability and its predictors in adolescents is scare. Suicide probability has been defined as a general tendency to kill oneself; it represents a novel measure for conceptualizing and assessing suicide risk. This construct is operationally defined by patients' scores on subscales such as suicide ideation, negative selfevaluation, hostility-impulsivity and hopelessness (4).

Non-suicidal self-injury (NSSI) is defined as the direct, deliberate destruction of one's own body without suicidal intent (5). Although suicidal attempts differ from NSSI, some studies show that NSSI can predict suicidal attempts over a six-month period (6). Previous findings have indicated that adolescents with NSSI and suicidal attempts, compared with NSSI alone, had higher scores in some of the suicide probability indices, such as impulsivity and suicide ideation $(7,8)$. Identifying specific factors that help to distinguish adolescents with NSSI alone, suicidal attempts alone, and combined forms of NSSI and suicidal attempts in terms of suicide probability, can help to develop better classifications for these two behaviors. So, one of the purposes of this study was to compare these groups in terms of suicide probability.

Although NSSI is associated with suicidal attempts, it seems this association is due to several underlying psychological risk factors. It has been identified that depression, a past history of suicidal attempts, low levels of social support, feelings of abandonment, and a history of sex- 
ual abuse are associated with suicidal attempts in adolescents (9). It would be useful to investigate other fundamental risk factors, such as emotional regulation difficulties and self-criticism, too. In other words, emotional regulation and self-criticism can be conceptualized as mechanisms or processes that link some known risk factors to suicidal attempts. Emotional regulation has been defined as people's methods of attending, evaluating, and modifying their emotional states (10). In a theoretical model, Gratz and Roemer (11) classified emotional regulation difficulties into six categories: the non-acceptance of emotional responses, difficulty in goal-directed behavior, impulse control difficulties, a lack of emotional awareness, limited access to emotion-regulation strategies, and a lack of emotional clarity. Theoretically, based on the cry of pain model of suicide (12), we can hypothesize that emotional regulation difficulties may be associated with suicide probability. Furthermore, adolescents with high impulsivity have been more prone to suicidal attempts (13). Previous findings have shown that a perceived lack of access to emotional regulation strategies was associated with suicide ideation and attempts in undergraduate students (14), but the relationship between emotion regulation difficulties and suicide probability in adolescents with a history of childhood maltreatment has not previously been studied.

Some researchers believe that emotion regulation difficulties play a common role in different kinds of children's and adults's psychopathologies (15). With this in mind, there are likely other risk factors beyond emotional dysregulation that increase the risk of suicide probability. One of these risk factors is the way people relate to their own selves. Self-criticism has been identified as a mediating risk factor that associated with suicidal attempts (16). In a novel formulation, self-criticism is defined as a feeling about the self. It can be classified as two forms: the inadequate self and the hated self (17). Previous studies have indicated these kinds of self-criticism are associated with suicidal attempts and NSSI (18). Experiencing trauma in childhood may disrupt the development of a healthy self and lead to pathological self processes like self-criticism; these processes could increase the risk of suicide. We hypothesized that different kinds of self-criticism could predict suicide probability after controlling for other risk factors, such as frequency of NSSI and difficulties in emotional regulation.

\section{Objectives}

The aims of this research were to compare suicide probability in adolescents with NSSI only, suicidal attempts and NSSI, and suicidal attempts only and to identify predictors of suicide probability based on NSSI, difficulties in emotion regulation, and forms of self-criticism.

\section{Patients and Methods}

We used a correlation and cross sectional design in this study. The sample consisted of 169 adolescents with a history of childhood maltreatment. They lived in Iranian social welfare centers in Tehran, Karaj, and Shahr-e Rey. These adolescents were all between the ages of 12 and 18 , and all can read and write. Having psychotic symptoms or cognitive deficits were exclusion criteria. According to Iranian social welfare centers' rules, all adolescents are screened to assessed for cognitive deficits and other psychotic symptoms before entering these social welfare institutions. Study subjects were 116 females and 53 males with an overall mean age of 14.23 years. All participants who enrolled in the study completed informed consent paperwork. When the results of tests showed a high risk of suicidal attempts, we informed the psychiatrist or other staff members of centers to plan a preventive intervention.

\subsection{Instruments}

\subsubsection{The Suicide Probability Scale}

The suicide probability scale consists of 36 statements. Participants are asked to rate every question based on a 4point Likert scoring system (ranging from "none or a little of the time" to "most or all of the time"). These ratings are then weighted selectively by item and summed to achieve a total weighted score and four subscale scores (4). Scale developers have reported an excellent internal consistency for the total scale, with an alpha coefficient of 0.93 , and internal consistency ranging from fair to good for the remaining scales (4). This scale was administered to Iranian adolescents, and the findings showed that scores on this scale can discriminate suicidal adolescents from nonsuicidal ones (19). In this study on adolescents with a history of childhood maltreatment, the alpha coefficient was obtained at 0.83 for the total scale.

\subsubsection{The Difficulties in Emotion Regulation Scale}

This scale examines six dimensions of emotion regulation skills. Respondents should indicate how often each item applies to them using a 5-point Likert scale ranging from 1 (almost never) to 5 (almost always). Previous studies have confirmed the factor structure and internal consistency of this scale in both foreign samples and among Iranian undergraduate students $(11,20)$. 
3.1.3. The Forms of Self-Criticizing/Attacking and SelfReassurance Scale

This scale consists of three subscales; we used two of them in this study: the inadequate self and the hated self. The scale starts with a general question, "When things go wrong for me..." and respondents rate 14 questions on a 5point scale (ranging from " 0 ," not at all like me, to “4," extremely like me). The factor structure of this scale in studies of university students has been confirmed, and the internal consistency of the scale was rated 0.88 for total sale (21). In our sample, factor analyses have confirmed the existence of two factors that explained 51\% of participants' scores on the scale $\left(\mathrm{KMO}=0.909, \chi^{2}=1.10, \mathrm{P}<0.001\right)$.

\subsubsection{The Non-Suicidal Self-Injury Checklist}

This checklist was used to assess the presence and frequency of NSSI in the past year. The checklist includes behaviors such as self-cutting, burning, hitting, head banging, rubbing, and picking at wounds (22) Participants are asked to indicate which one of these behaviors they have ever engaged in. Two additional questions were asked about the respondents' history of suicidal attempts and other self-harming behaviors not mentioned in the checklist. Questions were scored using a yes/no format. Participants were then classified as being a member of a group with NSSI, suicidal attempts, or both. A final question was asked to find the lifetime frequency of the NSSI.

\section{Results}

Among the 169 participants of this study, 116 (69\%) were female and 53 (31\%) were male. Descriptive results about NSSI methods and suicidal attempt in girls and boys are depicted in Table 1 . There were $41(24.4 \%)$ of participants between the ages of 12 and 14, 71 (42.3\%) were between the ages of 15 and 17, and 55 (32.7\%) were between the ages of 17 and 18 . The majority of the sample were secondary school students $(\mathrm{n}=77(46.2 \%))$, and $56(33.3 \%)$ were in high school grade $10,33(18.6 \%)$ were in grade 11 , and $3(1.5 \%)$ had their high school diploma. Participants lived in social welfare centers because of bad parenting and abuse, and we did not collect data on the family status of participants.

The most common method used for NSSI was selfcutting $\left(\chi^{2}=6.11, \mathrm{P}<00.1\right)$. The rate of self-cutting was significantly higher in girls than in boys $(P=0.017)$. The frequency of NSSI among participants include: $22(13 \%)$ one time, 12 (7\%) 2 - 4 times, 15 (9\%) 4 - 6 times, 6 (4\%) had engaged in NSSI more than 10 times, and 18 (11\%) do it often (more than 20 times).

Mean and standard deviation of groups with NSSI and suicidal attempt in suicide probability subscales are represented in Table 2. Among all participants, 69 (41.1\%) had a history of NSSI only, 48 (28.6\%) had a history of only suicidal attempts, while 52 (30.4\%) adolescents had a history of both suicidal attempts and NSSI. The frequency of suicidal attempts in the group with only suicidal attempts was one time, though there were two adolescents in this group who had attempted suicide 4 and 5 times. The frequency of suicidal attempts in the mixed group was between 1 and 5 times. The frequency of suicidal attempts in mixed group was higher than in the only suicidal attempt group.

There was a significant difference between groups with NSSI, suicidal attempts, and the mixed group in terms of self-criticism $(\mathrm{F}=14.62, \mathrm{df}=2,164 ; \mathrm{P}<0.001)$. The mean score of the mixed group $(M=22.35, S D=12.20)$ was more than the group with only NSSI $(M=12.15, S D=8.75)$ and group with only suicidal attempts $(M=19.96, S D=11.95)$.

A comparison between groups with NSSI, suicidal attempts, and both in suicide probability subscales with MANOVA (multivariate analysis of variance) showed a significant difference between groups (Wilkis lambda $=0.828$, $\mathrm{F}=2.27, \mathrm{P}=0.024)$. Analyses of variance showed a significant difference between the three groups on the suicide ideation subscale $(\mathrm{F}=3.46, \mathrm{P}=0.035)$. However, the group with suicidal attempts had the highest mean in suicide ideation, though the results of the post-hoc test (Dunnet test) showed that suicide ideation in the group with both behaviors (NSSI and suicidal attempts) was significantly higher than in the group with NSSI only $(\mathrm{P}<0.05)$.

The emotion regulation difficulties subscale includes a lack of emotional clarity, difficulty engaging in goaldirected behaviors, impulse control difficulties, and limited access to emotional regulation strategies; these were associated with suicide probability. The zero-order correlation is shown in Table 3.

A multiple regression analysis showed that the inadequate self, hated self, impulse control problems, and frequency of NSSI predicted suicide probability. This model can explain $50 \%$ of the variances of suicide probability. As presented in Table 4, the inadequate self, the hated self, impulse control problems, and frequency of NSSI, respectively, explained $40 \%, 5 \%, 3 \%$, and $2 \%$ of the variances of suicide probability. Results of the ANOVA showed this model was statistically significant $(\mathrm{F}=40.49, \mathrm{P}<0.001)$.

\section{Discussion}

The findings of this study show that suicide ideation in adolescents with a history of NSSI and suicidal attempts are significantly more common than in adolescents with NSSI alone. This finding is consistent with prior studies that compare suicidal attempt indicators in adolescents (6, 23). Unlike our results, which showed there was no significant difference between groups in terms of hostility- 
Table 1. Frequency of NSSI Methods and Suicidal Attempts by Gender

\begin{tabular}{|c|c|c|c|c|}
\hline & Female $^{a}$ & Male $^{\mathbf{a}}$ & Contingency Coefficient & P Value \\
\hline Cutting & $49(59)$ & $33(40)$ & 5.64 & 0.017 \\
\hline Burning & $21(63)$ & $12(36)$ & 0.058 & 0.45 \\
\hline Hitting & $43(65)$ & $23(34)$ & 0.083 & 0.56 \\
\hline Head banging & $38(64)$ & $21(35)$ & 0.074 & 0.338 \\
\hline Rubbing & $23(60)$ & $15(40)$ & 0.099 & 0.196 \\
\hline Picking wounds & $39(70)$ & $16(30)$ & 0.128 & 0.426 \\
\hline Suicidal attempt & $46(67)$ & $22(33)$ & 0.025 & 0.746 \\
\hline
\end{tabular}

${ }^{\mathrm{a}}$ Values are expressed as No. (\%).

Table 2. Mean and Standard Deviation of Groups on the Suicide Probability Subscale ${ }^{\mathrm{a}, \mathrm{b}}$

\begin{tabular}{lccc}
\hline & Group 1 & Group 2 & $24.9(3.8)$ \\
\hline Hopelessness & $24.7(5.4)$ & $25.9(5.7)$ & $20.5(5.1)$ \\
Hostility/impulsivity & $20.1(6.9)$ & $19.6(5.4)$ & $21.4(5.6)$ \\
Suicide ideation & $18.07(7.08)$ & $22.3(6.7)$ & $21.2(5.7)$ \\
Negative self-evaluation & $22.4(7.7)$ & $19.8(4.8)$ \\
\hline
\end{tabular}

${ }^{\text {a }}$ Descriptions: Group 1, NSSI only; group 2, NSSI + suicidal attempt; group 3, suicidal attempt.

${ }^{\mathrm{b}}$ Values are expressed as mean (\%).

Table 3. Zero-Order Correlation Between Difficulties in Emotion Regulation Subscales and Suicide Probability

\begin{tabular}{|c|c|c|c|c|c|c|c|}
\hline Variable & $\mathbf{1}$ & 2 & 3 & 4 & 5 & 6 & 7 \\
\hline 1-SP & - & & & & & & \\
\hline 2-Awarness & -0.093 & - & & & & & \\
\hline 3-Clarity & 0.383 & -0.003 & - & & & & \\
\hline 4-Nonaccept & 0.364 & -0.177 & -0.333 & - & & & \\
\hline 5-Impulse & 0.448 & 0.365 & 0.365 & 0.537 & - & & \\
\hline 6- Goals & 0.420 & -0.021 & -0.152 & 0.667 & 0.72 & - & \\
\hline 7-Strategies & 0.519 & -0.076 & 0.389 & 0.640 & 0.69 & 0.6 & - \\
\hline
\end{tabular}

Abbreviation: SP, suicide probability.

Table 4. Predictors of Suicide Probability by Emotion Regulation Difficulties, Forms of Self-Criticism and NSSI Frequency

\begin{tabular}{|c|c|c|c|c|c|c|}
\hline Variable & $\mathbf{R}$ & $\mathbf{R}^{2}$ & B & Beta & $\mathbf{t}$ & Sig \\
\hline Inadequate self & 0.63 & 0.407 & 0.580 & 0.249 & 2.62 & 0.009 \\
\hline Hated self & 0.67 & 0.454 & 1.50 & 0.337 & 3.72 & 0.001 \\
\hline Impulse control & 0.698 & 0.488 & 0.549 & 0.169 & 2.68 & 0.008 \\
\hline NSSI & 0.708 & 0.501 & 1.36 & 0.133 & 2.11 & 0.036 \\
\hline
\end{tabular}

impulsivity and hopelessness, another research on adolescents from a psychiatric inpatient unit in the United States revealed that adolescents with NSSI and suicidal attempt were more hopeless and have higher scores in impulsivity (24). This can be attributed to the setting of the studies and different scales used for assessing suicide probabil- 
ity indices. With this in mind, it can be concluded that the most reliable indicator of suicide probability is the experience of suicidal ideation. The findings of studies in adults in inpatient settings have revealed that suicidal thoughts remain relatively stable even after patients are released from the hospital (25), so clinicians who work with adolescents who have a history of suicidal attempts should consider the risk of suicide and apply preventive programs to educate these adolescents in ways of coping with suicide ideation. It should be noted that, based on the results of the present study, adolescents with a history of suicidal attempts had the highest score in all of the suicidal probability subscales, but this difference was not significant. Suicide probability of adolescents with suicidal attempts and NSSI was greater than in the NSSI-only group, but the suicide probability of adolescents with both suicidal attempts and NSSI was less than in the group with past suicidal attempts only. These findings are consistent with previous studies (26) that have indicated that adolescents with past suicidal attempts are more prone to subsequent suicide behaviors. Additionally, as people's experience with death cues, such as blood or the pain of self-injury increase, they become more prone to suicide risk (27).

A regression model indicated that an inadequate self, hated self, difficulty in impulse control and frequency of NSSI predicts a 50\% variance of suicide probability. Our results were consistent with a previous finding (16) that confirmed the role of self-criticism as a main risk factor for engaging in suicide behaviors (16). Some forms of selfcriticism, such as the inadequate self have greater association with suicide probability. According to the evolutionary model of self-criticism (18), self-criticism triggers threat and defensive emotions and behaviors, which lead to negative emotions that are difficult to regulate; thus, different kinds of psychopathology, like suicide behaviors, occur.

Although a zero-order correlation has revealed a significant association between difficulties in emotion regulation skills and suicide probability (except the difficulty in awareness subscale), in the regression model, only impulse control problems remained a significant predictor of suicide probability. This finding especially supports prior findings that showed impulsive adolescents are more inclined to suicidal attempts without prior planning (28). Our results did not confirm previous findings about the relationship between difficulties in emotion regulation skills and suicide ideation and behaviors (14). According to a previous study (14) of the emotion regulation subscale, only the perceived inability to access emotion regulation strategies predicted suicide ideation. It should be noted that we used a global index of suicide probability that consisted of ideation and inclination to suicidal at- tempts, rather than merely suicide ideation. So, it could be concluded that adolescents who have difficulty in controlling their impulses were more prone to suicide ideation and attempts.

Another predictor of suicide probability in the present study was the frequency of NSSI. This finding support previous studies, which have reported a significant association between NSSI and the tendency to suicide ideation and behaviors (6). However, it does not mean that every kind of NSSI leads to an increase in suicide probability. There have been different kinds of adolescents who engage in NSSI. Many of them engage NSSI just a few times, but a minority of adolescents who frequently use NSSI exclusively as a coping mechanism against any kind of life distress were more prone to act on their suicide ideations (28). Additionally, the association between NSSI and suicide probability can be explained based on Joiner's interpersonal theory of suicide (26). According to this theory, burdensome feelings and a loss of social belonging leads to suicide ideation, but the most determining factor for acting on suicide ideation is the beginning of harming oneself and decreasing fear of suicide. The persons who habituate to different kinds of self-harm are more capable of acting on suicide ideations. It can be concluded that increasing frequency of NSSI leads to suicide probability by acquiring the ability to engage fearlessly in lethal self-harm.

In sum, the present study indicates that adolescents with a history of NSSI and suicidal attempts, compared to a NSSI-only group, were more prone to subsequent suicidal attempts, because they have more suicide ideations, even after passing several years without engaging in a suicidal attempt. Additionally, our findings revealed that selfcriticism, impulse control difficulties, and the frequency of NSSI can predict suicide probability in adolescents with a history of childhood maltreatment. It should be noted that this study had a number of limitations. First, the design of this study was cross-sectional, and only self-report instruments were used for assessing our variables. Second, these findings were based exclusively on adolescents who had a history of childhood maltreatment, so they cannot be generalized to other adolescents in normal population or inpatient setting. Future studies should consider the role of these predictors of suicide probability while controlling for other known risk factors for suicidal attempts.

Adolescents with a history of childhood maltreatment, even after acute phases of suicide and hospitalization, are more prone to subsequent suicidal thoughts or behaviors. The risk of subsequent suicidal attempts is higher in adolescents with suicidal attempts and NSSI than in adolescents with NSSI only. Negative feelings about the self, like feelings of inadequacy or self-hatred, were a very important risk factor that can predict a great deal of variance in 
suicide probability. This study also showed that, among six dimensions of difficulties in emotion regulation skills (11), impulse control problems were a significant and stable predictor of suicidal attempts. So, psychological interventions should address impulse control problems in programs designed to prevent suicidal attempts. Adolescents who used NSSI frequently also are more prone to suicidal attempts. This association may be caused by their habituation to self-harming, which increases the risk of lethal self-harm. Indeed, according to the results of this study, it could be concluded that accepting one's self, instead of criticizing the self and overcoming impulse control problems, can decrease rates of suicide probability in adolescents with a history of childhood maltreatment.

\section{Acknowledgments}

All authors thank all of the study participants.

\section{Footnotes}

Authors' Contribution: Hamid Khanipour developed the original idea and protocol for the study, abstracted and analyzed the data, and wrote the manuscript. Mitra Hakim Shooshtari and Reza Bidaki contributed to the development of the protocol, abstracted data, and prepared the manuscript. Reza Bidaki also revised the manuscript.

Funding/Support: This research had not been sponsored by any organization or university.

\section{References}

1. Minino AM. Death in the United states, 2009. NCHS data Brief, 64 Hyattsville,: National Center for Health Statistics, Center for Disease Control and Prevention; 2009. Available from: http://www.cdc.gov/ nchs/nhis.htm

2. Nojomi M, Malakouti SK, Bolhari J, Poshtmashhadi M. A predictor model for suicide attempt: evidence from a population-based study. Arch Iran Med. 2007;10(4):452-8. [PubMed: 17903049].

3. Santa Mina EE, Gallop RM. Childhood sexual and physical abuse and adult self-harm and suicidal behaviour: a literature review. Can J Psychiatry. 1998;43(8):793-800. [PubMed: 9806085].

4. Cull JG, Gill W. Suicide probability scale. Los Angeles, CA: Western Psychological Services; 1982.

5. Nock MK, Prinstein MJ. A functional approach to the assessment of self-mutilative behavior.J Consult Clin Psychol. 2004;72(5):885-90. doi: 10.1037/0022-006X.72.5.885. [PubMed: 15482046].

6. Muehlenkamp JJ, Gutierrez PM. Risk for suicide attempts among adolescents who engage in non-suicidal self-injury. Arch Suicide Res. 2007;11(1):69-82. doi: 10.1080/13811110600992902. [PubMed: 17178643].

7. Brausch AM, Gutierrez PM. Differences in non-suicidal self-injury and suicide attempts in adolescents. J Youth Adolesc. 2010;39(3):233-42. doi:10.1007/s10964-009-9482-0. [PubMed: 19941045].

8. Cloutier P, Martin J, Kennedy A, Nixon MK, Muehlenkamp JJ. Characteristics and co-occurrence of adolescent non-suicidal self-injury and suicidal behaviours in pediatric emergency crisis services.J Youth Adolesc. 2010;39(3):259-69. doi: 10.1007/s10964-009-9465-1. [PubMed: 19856090].
9. Piqtkowski K, Sauer P. [Application of computer-assisted surgery (CAS) in otolaryngology-head and neck surgery]. Otolaryngol Pol. 2004;58(5):1027-32. [PubMed: 15732797].

10. Gross JJ. The emerging field of emotion regulation: an integrative review. Rev gen psychol. 1998;2(3):271.

11. Gratz KL, Roemer L. Multidimensional assessment of emotion regulation and dysregulation: development, factor structure, and initial validation of the difficulties in emotion regulation scale. J Psychopathol Behav Assess. 2004;26(1):41-54. doi: 10.1023/b:joba.0000007455.08539.94.

12. Williams JMG, Williams M. Cry of pain: Understanding suicide and self-harm. USA: Penguin Group; 1997.

13. Fergusson DM, Woodward LJ, Horwood LJ. Risk factors and life processes associated with the onset of suicidal behaviour during adolescence and early adulthood. Psychol Med. 2000;30(1):23-39. [PubMed: 10722173].

14. Oliva J. Retooling today's practices to accommodate part-time physicians. Physician Exec. 2005;31(1):56-9. [PubMed: 15732819].

15. Aldao A, Nolen-Hoeksema S, Schweizer S. Emotion-regulation strategies across psychopathology: A meta-analytic review. Clin Psychol Rev 2010;30(2):217-37. doi: 10.1016/j.cpr.2009.11.004. [PubMed: 20015584].

16. O'Connor RC, Noyce R. Personality and cognitive processes: self-criticism and different types of rumination as predictors of suicidal ideation. Behav Res Ther. 2008;46(3):392-401. doi: 10.1016/j.brat.2008.01.007. [PubMed: 18308293].

17. Gilbert P, Clarke M, Hempel S, Miles JN, Irons C. Criticizing and reassuring oneself: An exploration of forms, styles and reasons in female students. Br J Clin Psychol. 2004;43(Pt 1):31-50. doi: 10.1348/014466504772812959. [PubMed: 15005905].

18. Gilbert P, McEwan K, Irons C, Bhundia R, Christie R, Broomhead C, et al. Self-harm in a mixed clinical population: the roles of self-criticism, shame, and social rank. Br J Clin Psychol. 2010;49(Pt 4):563-76. doi: 10.1348/014466509X479771. [PubMed: 20109278].

19. Sharifian MS, Lavasani MG, Ejei J, Taremian F, Amrai K. The relationship among classroom community, attitude toward parents, anxiety disorders and depression with adolescent suicide probability. Procedia - Soc Behav Sci. 2011;15:520-5. doi: 10.1016/j.sbspro.2011.03.134.

20. Khanzadeh M, Saeediyan M, Hosseinchari M, Edrissi F. Factor structure and psychometric properties of difficulties in emotional regulation scale [Persian]. J behav sci. 2012;6(1):87-96.

21. Kupeli N, Chilcot J, Schmidt UH, Campbell IC, Troop NA. A confirmatory factor analysis and validation of the forms of selfcriticism/reassurance scale. Br J Clin Psychol. 2013;52(1):12-25. doi: 10.1111/j.2044-8260.2012.02042.x. [PubMed: 23398109].

22. Sansone RA, Wiederman MW, Sansone LA. The Self-Harm Inventory (SHI): development of a scale for identifying self-destructive behaviors and borderline personality disorder.JClin Psychol. 1998;54(7):97383. [PubMed: 9811134].

23. Dougherty DM, Mathias CW, Marsh-Richard DM, Prevette KN, Dawes MA, Hatzis ES, et al. Impulsivity and clinical symptoms among adolescents with non-suicidal self-injury with or without attempted suicide. Psychiatry Res. 2009;169(1):22-7. doi: 10.1016/j.psychres.2008.06.011. [PubMed: 19631392].

24. Qin P, Nordentoft M. Suicide risk in relation to psychiatric hospitalization: evidence based on longitudinal registers. Arch Gen Psychiatry. 2005;62(4):427-32. doi: 10.1001/archpsyc.62.4.427. [PubMed: 15809410].

25. Lewinsohn PM, Rohde P, Seeley JR. Psychosocial risk factors for future adolescent suicide attempts.JConsult Clin Psychol. 1994;62(2):297-305 [PubMed: 8201067].

26. Joiner TE. Why people die by suicide. Cambridge, MA: Harvard University Press; 2005. 
27. Witte TK, Merrill KA, Stellrecht NE, Bernert RA, Hollar DL, Schatschneider $\mathrm{C}$, et al. "Impulsive" youth suicide attempters are not necessarily all that impulsive. J Affect Disord. 2008;107(1-3):107-16. doi: 10.1016/j.jad.2007.08.010. [PubMed: 17804082].
28. Klonsky ED, Olino TM. Identifying clinically distinct subgroups of self-injurers among young adults: a latent class analysis. J Consult Clin Psychol. 2008;76(1):22-7. doi: 10.1037/0022-006X.76.1.22. [PubMed: 18229979]. 